\title{
Quantitative Thermal Microscopy Measurement with Thermal Probe Driven by dc+ac Current
}

\author{
Jerzy Bodzenta ${ }^{1}$ • Justyna Juszczyk ${ }^{1}$. \\ Anna Kaźmierczak-Bałata ${ }^{1}$. Piotr Firek ${ }^{2}$. \\ Austin Fleming $^{3}$ - Mihai Chirtoc ${ }^{4}$
}

Received: 13 October 2015 / Accepted: 14 May 2016 / Published online: 31 May 2016 (C) The Author(s) 2016. This article is published with open access at Springerlink.com

\begin{abstract}
Quantitative thermal measurements with spatial resolution allowing the examination of objects of submicron dimensions are still a challenging task. The quantity of methods providing spatial resolution better than $100 \mathrm{~nm}$ is very limited. One of them is scanning thermal microscopy (SThM). This method is a variant of atomic force microscopy which uses a probe equipped with a temperature sensor near the apex. Depending on the sensor current, either the temperature or the thermal conductivity distribution at the sample surface can be measured. However, like all microscopy methods, the SThM gives only qualitative information. Quantitative measuring methods using SThM equipment are still under development. In this paper, a method based on simultaneous registration of the static and the dynamic electrical resistances of the probe driven by the sum of dc and ac currents, and examples of its applications are described. Special attention is paid to the investigation of thin films deposited on thick substrates. The influence of substrate thermal properties on the measured signal and its dependence on thin film thermal conductivity and film thick-
\end{abstract}

This article is part of the selected papers presented at the 18th International Conference on Photoacoustic and Photothermal Phenomena.

$凶 \quad$ Justyna Juszczyk

justyna.juszczyk@polsl.pl

1 Institute of Physics, Silesian University of Technology, Konarskiego 22 B, 44-100 Gliwice, Poland

2 Institute of Microelectronics and Optoelectronics, Warsaw University of Technology, Koszykowa 75, 00-662 Warszawa, Poland

3 Mechanical and Aerospace Engineering Department, Utah State University, 4130 Old Main Hill, Logan, UT 84322-4130, USA

4 Multiscale Thermophysics Lab GRESPI-CATHERM, Université de Reims Champagne-Ardenne URCA, Moulin de la Housse, BP 1039, 51687 Reims, France 
ness are analyzed. It is shown that in the case where layer thicknesses are comparable or smaller than the probe-sample contact diameter, a correction procedure is required to obtain actual thermal conductivity of the layer. Experimental results obtained for thin $\mathrm{SiO}_{2}$ and $\mathrm{BaTiO}_{3}$ layers with thicknesses in the range from $11 \mathrm{~nm}$ to $100 \mathrm{~nm}$ are correctly confirmed with this approach.

Keywords Finite element method - Numerical analysis · Scanning thermal microscopy $\cdot$ Thermal conductivity measurement $\cdot$ Thin layers

\section{Introduction}

Rapid progress in the manufacturing of various types of structures and devices at micro- and nanometer scales, such as nanocomposites, superlattices, microelectronic, and optoelectronic devices, creates the need for a thorough understanding of the submicron-scale thermal transport mechanisms. For the vast majority of such devices, thermal management, thermal performance and efficiency of heat dissipation are important issues regarding the design and proper functioning as well as main factors restraining the progressive process of miniaturization.

Among many micro- and nanostructures, thin layers have attracted considerable attention due to their wide range of possible applications in e.g., microelectronics, data storage devices, or multilayer coatings. Thermal properties of thin films have been extensively investigated for a variety of materials [1-5] using different techniques, e.g., the $3 \omega$ method [6] and the thermo-reflectance technique [7-9]. The thermal conductivity of thin films may be considerably lower than that of bulk materials and for most of them a decrease of the thermal conductivity is observed with a decrease in the layer thickness [10-12]. This is mainly due to structural defects or interfacial boundaries introducing additional thermal resistance and acting as potential scattering centers for heat carriers (electrons in metals and phonons in dielectrics). In the case of very thin films, the restriction of phonon and electron heat transport can also be caused by reduction of the number of available dimensions. The mean free path (MFP) for electrons in metals at room temperature is about $10 \mathrm{~nm}$, and for phonons in the dielectrics and semiconductors is in the range between $10 \mathrm{~nm}$ and $100 \mathrm{~nm}$ [13]. If the structure size becomes comparable to or smaller than the MFP of energy carriers, processes connected with the heat transport become qualitatively different from those in the macroscopic systems and effects associated with the wave nature of heat carriers emerge.

In classical thermodynamics, thermal transport is described by Fourier's law and the validity of its application determines the boundary between the classical and nanoscale description of heat transport. In nanoscale regime, sub-continuum effects need to be considered, thus description based on continuum heat transport equations is no longer valid [11].

In theoretical descriptions of heat transfer in nanoscale, most approaches are based on numerically solving the Boltzmann transport equation (BTE) $[14,15]$ or on molecular dynamics simulations $[16,17]$. While the dimensions of the structure reach scales comparable to the MFP of heat carriers, a transition of heat transport mechanism from diffusive to ballistic occurs, which has been widely investigated for various types of 
structures [14,18-22]. The dependence of effective thermal conductivity, $\kappa$, on structure size can be described by the equation [23-25]:

$$
\kappa(L)=\frac{\kappa_{0} L^{2}}{2 \pi^{2} \lambda^{2}}\left[\sqrt{1+4\left(\frac{\pi \lambda}{L}\right)^{2}}-1\right],
$$

where $L$ is the characteristic size of the structure (e.g., the thickness of the layer), $\lambda$ is the MFP of heat carriers, and $\kappa_{0}$ is the thermal conductivity of a bulk material. When $\lambda \ll L, \kappa$ tends to $\kappa_{0}$, and its value does not depend on the size. The heat transport has diffusive character and can be described by the Fourier's law. When $\lambda \gg L$, the ballistic heat transport occurs and $\kappa$ depends linearly on $L$. In this case, the heat flux does not depend on the temperature gradient but on the temperature difference $[23,25]$.

As the thermal conductivity changes with the thickness of the layer and depends on layer inner structure (e.g., grain size), it is very difficult to estimate it theoretically. The only possibility to determine the thermal conductivity is to measure it. However, standard measuring methods are not applicable for thin films. This is why development of new methods for investigating thermal properties that provide sufficient resolution is important. Scanning thermal microscopy (SThM) is a technique which can be used for investigation of thermal transport in nanoscale and determination of local thermal properties. It utilizes standard scanning probe microscope system equipped with a thermal module and a thermal probe. There are two standard SThM operation modes available. In the passive mode, the probe serves as a temperature sensor and a temperature distribution along sample surface is measured. In the active mode, the probe acts as a sample heater and the temperature sensor simultaneously, and a distribution of local thermal conductivity can be obtained [26,27]. The SThM allows highly localized measurements of thermal properties of micro- and nanostructures [28-30]. Nanofabricated thermal probes (NThP) provide spatial resolution better than $100 \mathrm{~nm}$ [31], and good temperature sensitivity. SThM has been used to study the thermal properties of various thin films, e.g., meso-porous silicon films [32,33], nanocrystalline diamond films [34], amorphous silicon films [35], or diamond-like nanocomposite films [36]. More information about the technique can be found in the SThM review article [37]. In the case of bulk materials or relatively thick samples, the thermal conductivity is usually determined from calibration curve obtained from SThM measurements performed for reference samples of known thermal properties. Measurements can be carried out in different SThM operation modes. Selected examples can be found in Refs [38-41]. The method for quantitative SThM measurement based on simultaneous determination of the static and dynamic NThP resistances was proposed by a few authors of this paper. Details of the method are given in Sect. 1.

In the case of a very thin film, the SThM signal is influenced not only by the thermal properties of the film, but also by the thermal properties of the substrate. It is justified to assume, that the influence of the substrate should be taken into account for films of thicknesses smaller than, or comparable to the depth resolution of the SThM probe $[32,33]$. This resolution is estimated to be at most a few times the range of the radius of the probe-sample contact [32], which in case of NThP is of the order of $100 \mathrm{~nm}$. The problem of measuring the thermal properties of thin films using SThM and the corre- 
lation between the effective thermal conductivity (or thermal resistance) of a thin layer and layer thickness have been investigated e.g., in [12,42]. One of the major problems associated with the use of SThM for quantitative measurements is the lack of a satisfactory model describing the processes occurring in the probe-sample system. The complex geometry of the probe, especially the NThP, impedes the creation of proper analytical description. In this case, an interesting tool for the analysis of processes in the probe-sample system is numerical simulation based on the finite element method (FEM) [43-47]. So far, we have presented the probe-sample FEM model $[39,48]$ which allowed accurate modeling of the basic physical processes occurring during the SThM measurement. It enabled the estimation of the dynamic range of signal changes and determining the highest sensitivity regime. In this work, we present the results of numerical simulations of SThM measurement on a layered sample. The influence of the substrate thermal conductivity and thickness as well as the layer thermal conductivity on the SThM signal is investigated. The layer thicknesses varied between 0.01 $\mu \mathrm{m}$ and $100 \mu \mathrm{m}$. Based on the obtained results, a general procedure was proposed which allows for the correction of the apparent thermal conductivity value obtained directly from the experiment by taking into account the thickness of the investigated layer and the thermal conductivity of substrate. Experimental results carried out for selected thin layers complement the analysis.

\section{Quantitative Scanning Thermal Microscopy with dc+ac-Driven Thermal Probe}

As mentioned in the Introduction, SThM can give information about local temperature (in passive mode), or local thermal conductivity (in active mode) of the sample. Quantitative thermal measurement with SThM equipment is accomplished by relatively low sensitivity of SThM signal to variations of thermal conductivity of the sample. The thermal model of NThP, which allows analysis of the sample thermal conductivity on the SThM signal, was proposed in Ref. [49]. A probe-sample system was modeled using electrical analogies of heat flows. It was shown that the sample thermal conductivity $\kappa$ influences the measured signal through the constriction thermal resistance

$$
R=\frac{1}{4 \kappa r}
$$

where $r$ is the probe-sample contact radius. However, this thermal resistance is connected in series with the thermal resistance of the physical contact between probe and sample, and the constriction thermal resistance of the NThP. It makes absolute determination of $R$ difficult. Moreover, the main heat flux from heated probe region flows through the probe cantilever to the probe mount. Because of these two facts, SThM signal changes caused by changes of the thermal conductivity of the sample do not exceed a few per cent.

The absolute value of the signal changes can be increased by increasing the heat dissipated in the NThP. However, two limitations must be taken into account. According to the probe specification given by the manufacturer, the maximum dc probe current 
for NThP is limited to about $2.2 \mathrm{~mA}$. However, starting from $1.8 \mathrm{~mA}$ to $1.9 \mathrm{~mA}$ probe degradation was observed. For currents of about $1.7 \mathrm{~mA}$ the SThM signal changes are low and must be detected on relatively high dc background. A possible solution is driving the NThP with ac current. Such technique combined with third harmonic detection is popular for Wollaston wire thermal probes. But in the case of NThPs, periodical probe heating causes periodical probe bending, which affects mechanical stability of the probe and makes the measurement difficult or even impossible. Such effect is not present in case of dc techniques, however, in this case, the sensitivity to the thermal properties is low. The solution to these problems was proposed in Ref. [50]. The idea was to make use of good points of $\mathrm{dc}$ and ac experiments. DC techniques assure mechanical stability of the probe-sample system, while ac techniques allows improvement of signal-to-noise ratio thanks to lock-in signal detection. In the mentioned paper, we proposed the technique based on measurement with dc-biased NThP with small ac component superimposed. It was shown that the ac component of the probe voltage is three times more sensitive to the changes of the sample thermal conductivity than the dc one. It is also important that information about the sample thermal properties can be obtained likewise from the phase shift of the ac component. Moreover, the ratio $\frac{\left(R_{\mathrm{d}}-R_{\mathrm{S}}\right)_{\text {in }}}{\left(R_{\mathrm{d}}-R_{\mathrm{S}}\right)_{\text {out }}}$ (where $R_{\mathrm{S}}$ and $R_{\mathrm{d}}$ are static and dynamic NThP electrical probe resistances, and subscript "in" stands for measurement done for NThP in contact with the sample, and subscript "out"-for the NThP in air) measured at low frequency is equal to the ratio of effective thermal resistances to the heat transport from the probe to its surrounding in mentioned cases. This ratio depends on the sample thermal conductivity and is immune to environmental conditions.

The usefulness of the proposed technique was proved experimentally. In the next Section, the problem of determination of the thermal conductivity of thin films is analyzed.

\section{Numerical Analysis}

As mentioned in the previous section, to obtain the actual value of thermal conductivity of the film in the case of film thicknesses comparable with the diameter of the probesample contact, the influence of the thermal properties of the film substrate must be taken into account. The analysis of this influence is presented in this section. This analysis was carried out based on finite element model of the probe-sample system.

The geometry of the electro-thermal finite element model of the probe-sample system, created in COMSOL Multiphysics, is presented in Fig. 1.

A detailed description of the individual components of the model as well as their geometrical and material parameters can be found in [39]. The sample is modeled by a hemisphere substrate with a flat layer which is in contact with the probe tip. The diameter of the probe-sample contact area is $100 \mathrm{~nm}$, which is the estimated value of the tip radius. The probe-sample contact is surrounded by air. By virtue of the symmetry of the system, only half of the probe and the sample are needed to be considered. The boundary and initial conditions set into the model were 
Fig. 1 The geometry of the probe-sample model (a). The sample consists of hemisphere substrate with a layer and is surrounded by air (b).

Magnification of the area of the probe-sample contact (c). The model consists of probe substrate-1, contacts-2, resistive strip - 3 , sample substrate -4 , thin layer- 5 , surrounding air- 6 , and contact layer-7
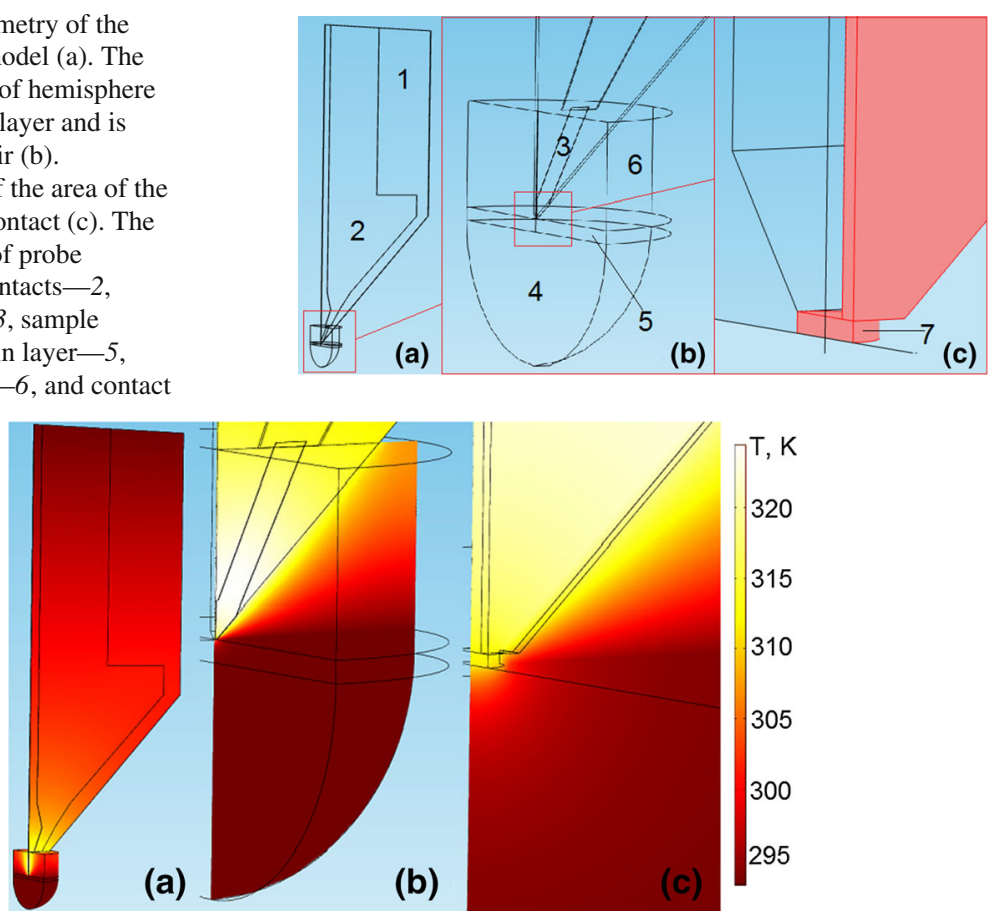

Fig. 2 An exemplary temperature distribution in the probe-sample system (a). Temperature distribution in the $x=0$ plane (the upper plane of the probe substrate) (b). Magnified area in the vicinity of the probesample contact (c). The calculations were made for a probe current of $1.5 \mathrm{~mA}, \kappa=10 \mathrm{~W} \cdot \mathrm{m}^{-1} \cdot \mathrm{K}^{-1}, \kappa_{0}=$ $100 \mathrm{~W} \cdot \mathrm{m}^{-1} \cdot \mathrm{K}^{-1}$, and $d=1 \mu \mathrm{m}$.

- temperature and the heat flux continuity assumed at all inner boundaries,

- thermal insulation and electrical grounding at the symmetry plane,

- convective cooling on remaining outer boundaries,

- constant electric current density through the outer cross section of the contact,

- initial temperature of $293.15 \mathrm{~K}$ and electric potential of $0 \mathrm{~V}$.

The thickness of the layer, $d$, and its thermal conductivity, $\kappa$, as well as the thermal conductivity of the substrate, $\kappa_{0}$, were the parameters of the simulation. The model allowed for the determination of the voltage drop on the probe heated by a constant current and being in thermal contact with the layered sample, as a function of those parameters. The probe current was $1.5 \mathrm{~mA}$. The probe signal was calculated for thin film thermal conductivity ranging from $0.5 \mathrm{~W} \cdot \mathrm{m}^{-1} \cdot \mathrm{K}^{-1}$ to $1000 \mathrm{~W} \cdot \mathrm{m}^{-1} \cdot \mathrm{K}^{-1}$ and thickness ranging from $10 \mathrm{~nm}$ to $100 \mu \mathrm{m}$. Simulations were performed for two thermal conductivities of the substrate: $10 \mathrm{~W} \cdot \mathrm{m}^{-1} \cdot \mathrm{K}^{-1}$ and $100 \mathrm{~W} \cdot \mathrm{m}^{-1} \cdot \mathrm{K}^{-1}$. These two values were chosen to simulate actual layers deposited on poor and well-conductive substrates, i.e., glass or silicone and to compare the influence of the substrate thermal properties on measured signal in both cases.

The exemplary temperature distribution in the probe-sample system calculated using presented model is presented in Fig. 2. 
Fig. 3 Normalized spreading thermal resistance of the layered sample as a function of dimensionless layer thermal conductivity $\kappa / \kappa_{0}$ and dimensionless layer thickness $d / a$.

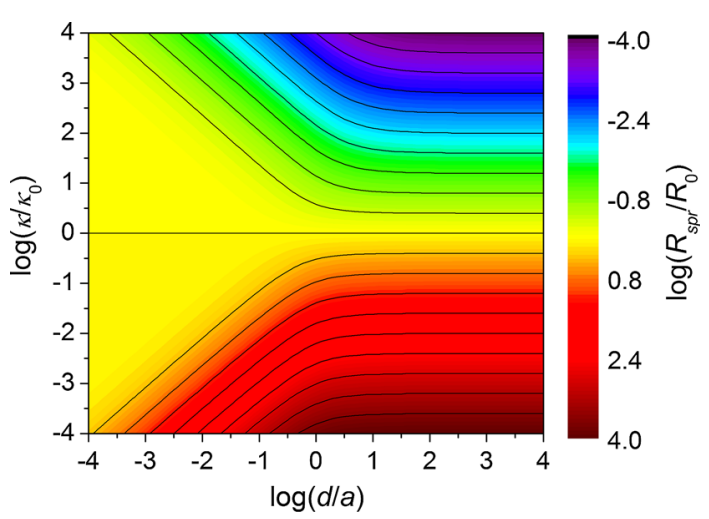

The highest temperatures are observed in the area of the resistive strip of the probe. The temperature disturbance penetrates the sample, however, noticeable temperature rise is observed only in the close vicinity of the probe-sample contact. Thus, it can be concluded that the probing depth is primarily determined by the diameter of the probesample contact. During active SThM experiments heat enters the sample through the circular probe-sample contact area. In the case of heat entering an isotropic half-space through circular area, the flux lines spread apart and the heat is conducted away from the source area. The thermal resistance in such case is called a spreading thermal resistance. Extensive thermal resistance analysis for a wide variety of cases can be found in [51]. The case of a thin layer on a substrate corresponds to a spreading thermal resistance of a half-space system containing a single layer on a substrate. For a circular source of radius $a$ in contact with an isotropic layer of thickness $d$ and thermal conductivity $\kappa$, which is in perfect thermal contact with an isotropic half-space of thermal conductivity $\kappa_{0}$, the normalized spreading resistance, i.e., the ratio of the spreading resistance of the layered system $R_{\text {spr }}$ to the one of the isotropic half-space $R_{0}$, can be expressed by the integral [51]

$$
\frac{R_{\mathrm{spr}}}{R_{0}}=\frac{4 \kappa_{0}}{\pi \kappa} \int_{0}^{\infty} \frac{\lambda_{2} \exp (\zeta d / a)+\lambda_{1} \exp (-\zeta d / a)}{\lambda_{2} \exp (\zeta d / a)-\lambda_{1} \exp (-\zeta d / a)} \cdot \frac{J_{1}(\zeta) \sin \zeta}{\zeta^{2}} d \zeta
$$

where $\lambda_{1}=\left(1-\kappa_{0} / \kappa\right), \lambda_{2}=\left(1+\kappa_{0} / \kappa\right)$, and parameter $\zeta$ is a dummy variable of integration. Normalized spreading resistances, calculated according to the Eq. 3 for a wide range of dimensionless layer thickness $d / a$ and layer thermal conductivity $\kappa / \kappa_{0}$ are presented in Fig. 3. The main conclusion which can be drawn from Fig. 3 is that the influence of the substrate on the total thermal spreading resistance of the system disappears for the layers thicker than a few source radii $a$. An exact thickness of the layer for which the influence of the substrate can be neglected depends on the layer thermal conductivity and is smaller for lower $\kappa$. The results showed in Fig. 3 confirm the statement that the temperature disturbance in analyzed system propagates on distances comparable with the source diameter.

The theoretical analysis based on Eq. 3 allows the estimation of the layer thickness for which the influence of the heat transport in the substrate on the spreading resistance 
of the system is negligible, and the layered sample can be treated as a homogeneous one. However, the effective thermal resistance depends on the spreading resistance of the probe-sample contact in a complex way. Detailed analysis can be found in [50]. Because of many parameters of the model, analytical analysis of the influence of $\kappa$, $\kappa_{0}$, and $d$ on measured signal is a complex task. This is why the analysis was carried out by the use of numerical model of the experiment. Presented numerical FEM model includes all elements that contribute to the total thermal resistance of the probe-sample system. The contact thermal resistance was taken into account by addition of a small cylindrical contact layer connecting the probe and sample, with a diameter of $100 \mathrm{~nm}$. Such a model allows the calculation of the probe signal in contact with a thin layer, which may be directly compared with the actual values obtained during the real experiment. In the frame of the quantitative analysis, the probe signal dependence on the thickness and the thermal conductivity of the layer was investigated for two thermal conductivities of the substrate. Figure 4 shows the relative probe voltage changes as a function of thermal conductivity ratio $\kappa / \kappa_{0}$ and the relative layer thickness $d / a$, calculated for the substrate thermal conductivities $\kappa_{0}=10 \mathrm{~W} \cdot \mathrm{m}^{-1} \cdot \mathrm{K}^{-1}$ (Fig. 4a) and $100 \mathrm{~W} \cdot \mathrm{m}^{-1} \cdot \mathrm{K}^{-1}$ (Fig. 4b). Comparing the results obtained by theoretical analysis and numerical simulations, it can be stated that changes of the thermal spreading resistance of the sample shown in Fig. 3, which are a few orders of magnitude, are much higher than the relative changes of the probe signal shown in Fig. 4, which are only a few percent. This is due to the fact that the spreading thermal resistance of the sample is only one component constituting the total thermal resistance of the probe-sample system, which determines the signal measured in the experiment. In contrast to the theoretical results presented in Fig. 3, which depends only on $\kappa / \kappa_{0}$ ratio, the probe voltage dependence on $\kappa / \kappa_{0}$ is different for different thermal conductivities of the substrate, as it is shown in Fig. 4c. Therefore, it must be calculated separately for each specific substrate.

The numerical data shown as a function of layer thickness for various layer thermal conductivities are presented in Fig. 5. It is clearly visible that the probe signal depends on the thickness and the thermal conductivity of the layer. The strongest influence of the layer thickness is observed for layers thinner than $1 \mu \mathrm{m}$. For layers thicker than $10 \mu \mathrm{m}$, the influence of the substrate thermal conductivity on the measured signal can be neglected. Moreover, the influence of the substrate on the result of SThM measurement depends on the thermal conductivity of the layer itself. For materials with low thermal conductivity, the influence of the substrate remains noticeable for thicknesses up to few $\mu \mathrm{m}$, while for well-conductive materials it can be neglected even for layers thinner than $1 \mu \mathrm{m}$. With decreasing thickness of the layer, the probe signal approaches the limit value corresponding to the thermal conductivity of the substrate. Similar behavior of SThM signal in case of different film and substrate thermal conductivities was predicted in [42].

The SThM signal dependence on the layer thickness $d$ can be described by empirical expression

$$
U(d)=U_{\mathrm{bulk}}+\frac{U_{\mathrm{sub}}-U_{\mathrm{bulk}}}{1+\frac{d}{d_{0}}},
$$


Fig. 4 Relative probe voltage changes, calculated on the basis of FEM model, as a function of the thermal conductivity ratio $\kappa / \kappa_{0}$ and the relative layer thickness $d / a$ for $\kappa_{0}=10$ $\mathrm{W} \cdot \mathrm{m}^{-1} \cdot \mathrm{K}^{-1}$ (a) and 100 $\mathrm{W} \cdot \mathrm{m}^{-1} \cdot \mathrm{K}^{-1}$ (b). Comparison of both signals for relative layer thickness $d / a=1$ (c)
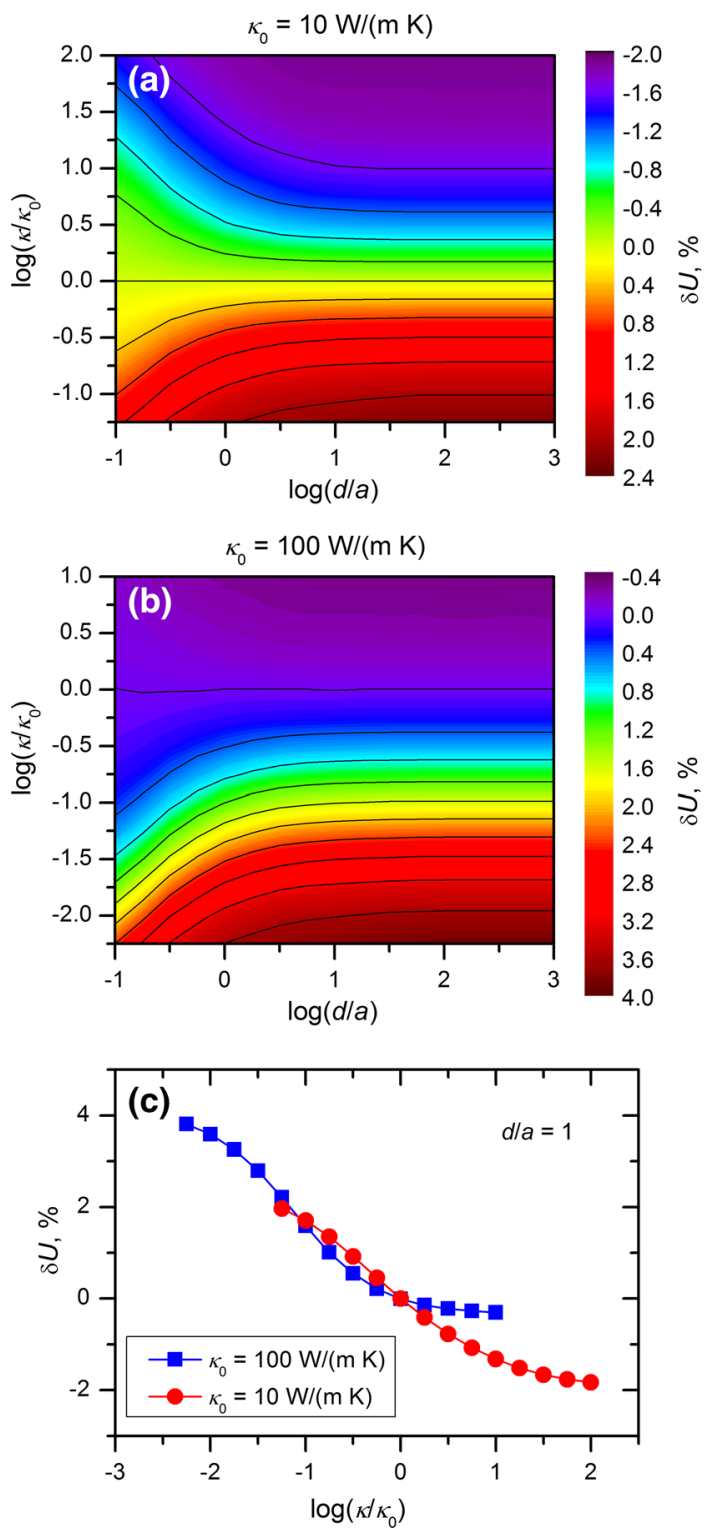

where $U_{\text {bulk }}$ and $U_{\text {sub }}$ are the probe signals for the homogeneous samples with the thermal conductivities $\kappa$ and $\kappa_{0}$, respectively, and $d$ is the layer thickness for which $U=\left(U_{\text {sub }}+U_{\text {bulk }}\right) / 2$. Equation (4) can be considered as the transfer function of the two-phase system. For very thin layers, it is expected that the probe signal will be approaching asymptotically to the value corresponding to the signal registered in contact with the substrate material. With increasing layer thickness, the probe signal approaches the value corresponding to the bulk sample. Solid lines in Fig. 5 are curves 

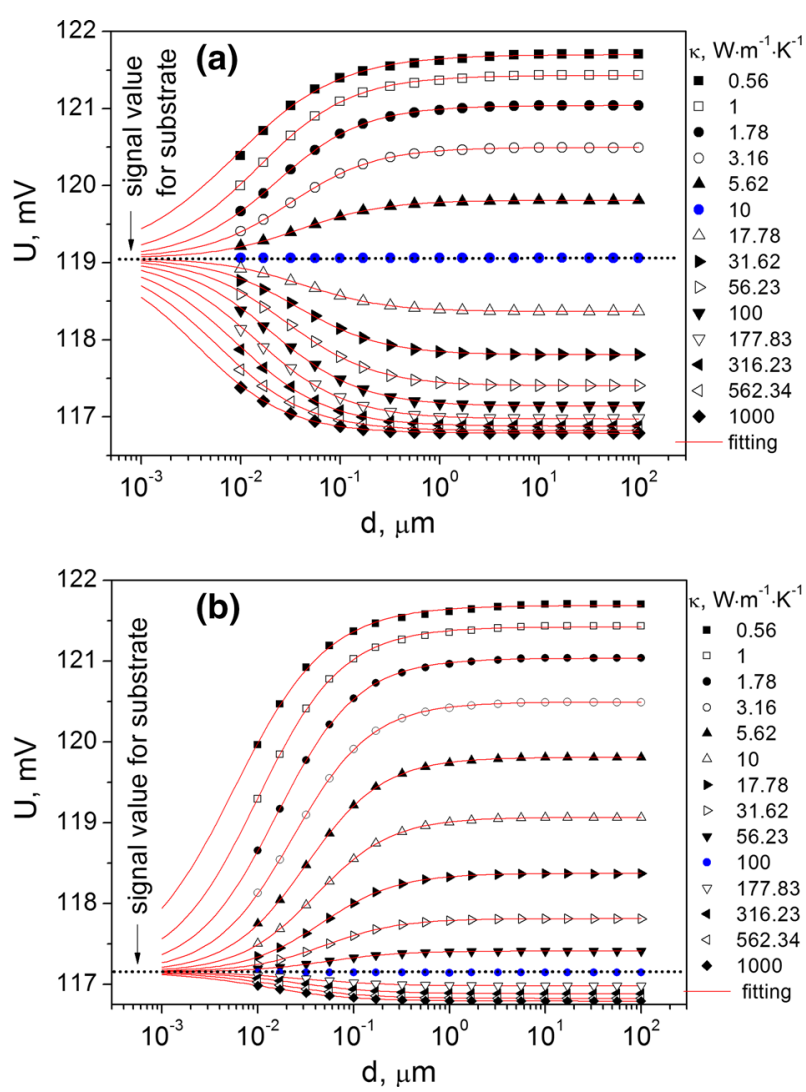

Fig. 5 Probe voltage as a function of thin layer thickness. Results of numerical simulations (points) and theoretical curve fitting (solid lines) for $\kappa_{0}=10 \mathrm{~W} \cdot \mathrm{m}^{-1} \cdot \mathrm{K}^{-1}$ (a) and $100 \mathrm{~W} \cdot \mathrm{m}^{-1} \cdot \mathrm{K}^{-1}$ (b)

described by Eq. 4 fitted to the numerical data. Figure 6 shows the dependence of the parameter $d_{0}$ on the thermal conductivity $\kappa$ of the layer for $\kappa_{0}=10 \mathrm{~W} \cdot \mathrm{m}^{-1} \cdot \mathrm{K}^{-1}$. It can be seen that this parameter approaches infinity for $\kappa \rightarrow \kappa_{0}$ and is smaller than $10 \mathrm{~nm}$ for $\kappa \gg \kappa_{\text {sub }}$ and $\kappa \ll \kappa_{0}$. The $d_{0}$ value can be used as a criterion of treating the layer as a bulk sample. For $d>10 d_{0}$, a difference between $U(\mathrm{~d})-U_{\text {sub }}$ and $U_{\text {bulk }}-U_{\text {sub }}$ is less than $10 \%$. Therefore, layers thicker than $10 d_{0}$ can be treated as bulk samples, and the influence of the substrate can be neglected.

The main goal of numerical analysis of the SThM measurement on layered samples was to develop a method which allows for the determination of a corrected thermal conductivity of the thin film from the apparent thermal conductivity obtained directly from the experiment. The method is based on calibration curves obtained from the numerical analysis. These curves were built using the SThM signal dependence on $\kappa$ and $d$ for given $\kappa_{0}$. Such curves obtained for $\kappa_{0}=100 \mathrm{~W} \cdot \mathrm{m}^{-1} \cdot \mathrm{K}^{-1}$ are shown in Fig. 7.

The principle of the calibration procedure is as follows. The apparent thermal conductivity of the sample is obtained from experimental data, based on calibration 
Fig. 6 Dependence of the parameter $d_{0}$ on the thermal conductivity of the layer for $\kappa_{0}=10$

Fig. 7 Correction of the results obtained directly from SThM measurement by calibration curves constructed on the basis of numerical simulations.

Exemplary contour plot created based on numerical data calculated for $\kappa_{0}=100$ $\mathrm{W} \cdot \mathrm{m}^{-1} \cdot \mathrm{K}^{-1}$
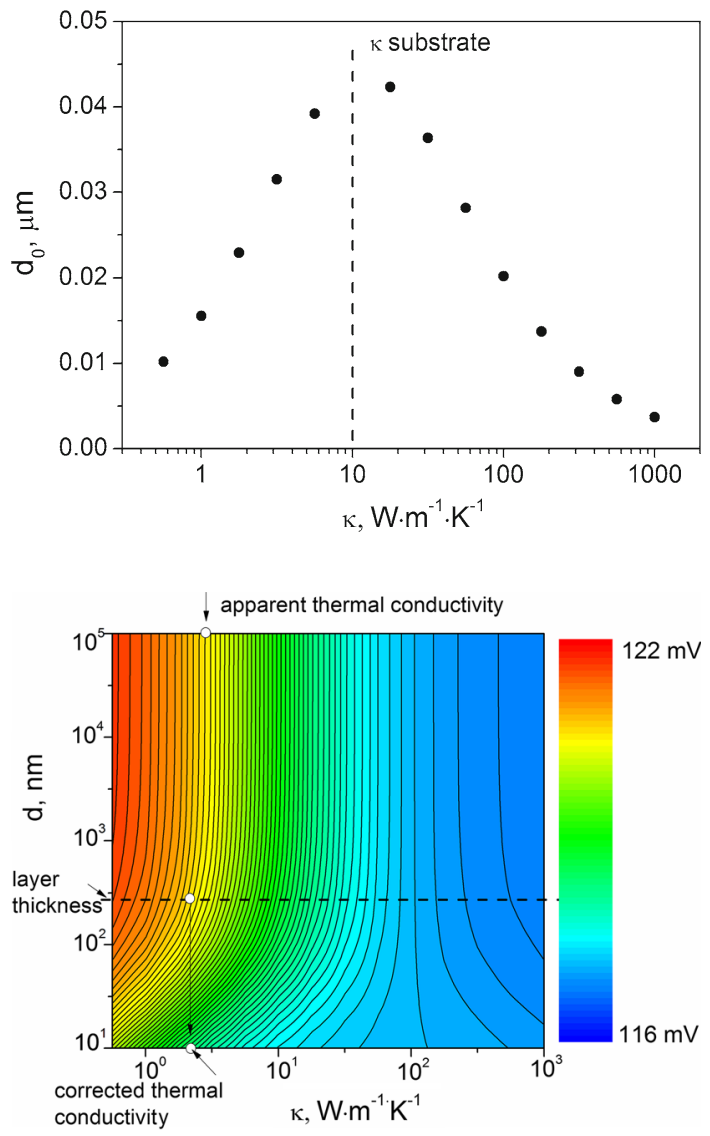

curve constructed from measurements performed for the reference samples of known thermal conductivities. The corrected value of the thermal conductivity is read from calibration curves based on numerical data. Starting from the point corresponding to the apparent thermal conductivity value, along the line in the direction of decreasing layer thickness, the corrected thermal conductivity value is read at the intersection point with the line drawn at the point corresponding to the layer thickness.

\section{Experimental Results and Discussion}

In the experimental part, SThM measurements were performed for reference samples of known thermal properties, two thin $\mathrm{SiO}_{2}$ samples and two thin $\mathrm{BaTiO}_{3}$ samples. The thermal conductivities of reference samples are presented in Table 1. Thin layers were prepared and characterized in the Institute of Microelectronics and Optoelectronics at Warsaw University of Technology. $\mathrm{SiO}_{2}$ thin layers were manufactured by dry thermal oxidation in a furnace with controlled ambient conditions fulfilling the Deal-Groove model assumptions of thermal oxide growth. The thicknesses of the $\mathrm{SiO}_{2}$ layers were 
Table 1 Reference samples used in the experiment and their thermal conductivity values

\begin{tabular}{ll}
\hline Sample & $\begin{array}{l}\text { Thermal conductivity } \\
\kappa, \mathrm{W} \cdot \mathrm{m}^{-1} \cdot \mathrm{K}^{-1}\end{array}$ \\
\hline $\mathrm{BK} 7$ & 1.0 \\
$\mathrm{YAG}$ & 10.0 \\
$\mathrm{Ti}$ & 21.9 \\
$\mathrm{GaSb}$ & 32 \\
$\mathrm{Si}$ & 140 \\
$\mathrm{Au}$ & 317 \\
$\mathrm{SiC}$ & 490 \\
\hline
\end{tabular}

$11 \mathrm{~nm}$ and $15 \mathrm{~nm}$. The $\mathrm{BaTiO}_{3}$ films were deposited on n-type Si substrates using the radio frequency plasma sputtering in argon atmosphere. The thickness of the layers are $100 \mathrm{~nm}$. One of the $\mathrm{BaTiO}_{3}$ samples was annealed after deposition for $30 \mathrm{~min}$ at $600{ }^{\circ} \mathrm{C}$ in argon atmosphere. Results for $\mathrm{BaTiO}_{3}$ layers have been partially published in Ref. [52]. The SThM measurements based on the determination of dynamic $R_{\mathrm{d}}$ and static $R_{\mathrm{S}}$ electrical resistances of the thermal probe being in contact with the sample and surrounding by air. Detailed description of the measurement technique and the correlation between the measured signal and thermal properties of the sample can be found in [50].

The SThM measurements were carried out using PSIA XE-70 scanning microscope equipped with the thermal module. A nanofabricated thermal probe KNT-SThM-1an (KELVIN Nanotechnology) was used. The probe was connected in series with a $10 \mathrm{k} \Omega$ balance resistor and driven with a sum of DC current of $1.5 \mathrm{~mA}$ and AC component of amplitude of $0.075 \mathrm{~mA}$ and frequency $320 \mathrm{~Hz}$. The probe electrical resistances were determined for the probe being in contact with the samples and probe lifted $2.0 \mathrm{~mm}$ above the sample surface. For each sample two measurement series were performed at three different points on the sample surface.

SThM measurements were performed for $\mathrm{SiO}_{2}$ and $\mathrm{BaTiO}_{3}$ thin layers and reference samples. The results are presented in Fig. 8. The solid circles represent the experimentally determined ratio $\frac{\left(R_{\mathrm{d}}-R_{\mathrm{S}}\right)_{\text {in }}}{\left(R_{\mathrm{d}}-R_{\mathrm{S}}\right)_{\text {out }}}$ for reference samples. Taking into account that the thermal resistance between the probe and its surroundings $R_{\text {th }}$ can be described by the expression $[39,50]$ :

$$
R_{\mathrm{th}}^{-1}=h+\left(R_{\mathrm{thP}}+\frac{1}{4 \kappa r}\right)^{-1}
$$

where $h$ is the effective heat transfer coefficient describing convective cooling of the probe, $R_{\text {thP }}$ is a sum the probe thermal resistance to the heat flux from the heat sources to the probe sample contact and the thermal resistance of the thermal barrier at the contact, $\kappa$ is the thermal conductivity of the sample, and $r$ is the probe-sample contact radius, the $\left.R_{\mathrm{th}}\right|_{\text {in }} /\left.R_{\mathrm{th}}\right|_{\text {out }}$ ratio dependence on $\kappa$ is

$$
\frac{\left.R_{\mathrm{th}}\right|_{\text {in }}}{\left.R_{\mathrm{th}}\right|_{\text {out }}} \approx \frac{1+A \kappa}{1+B \kappa}
$$



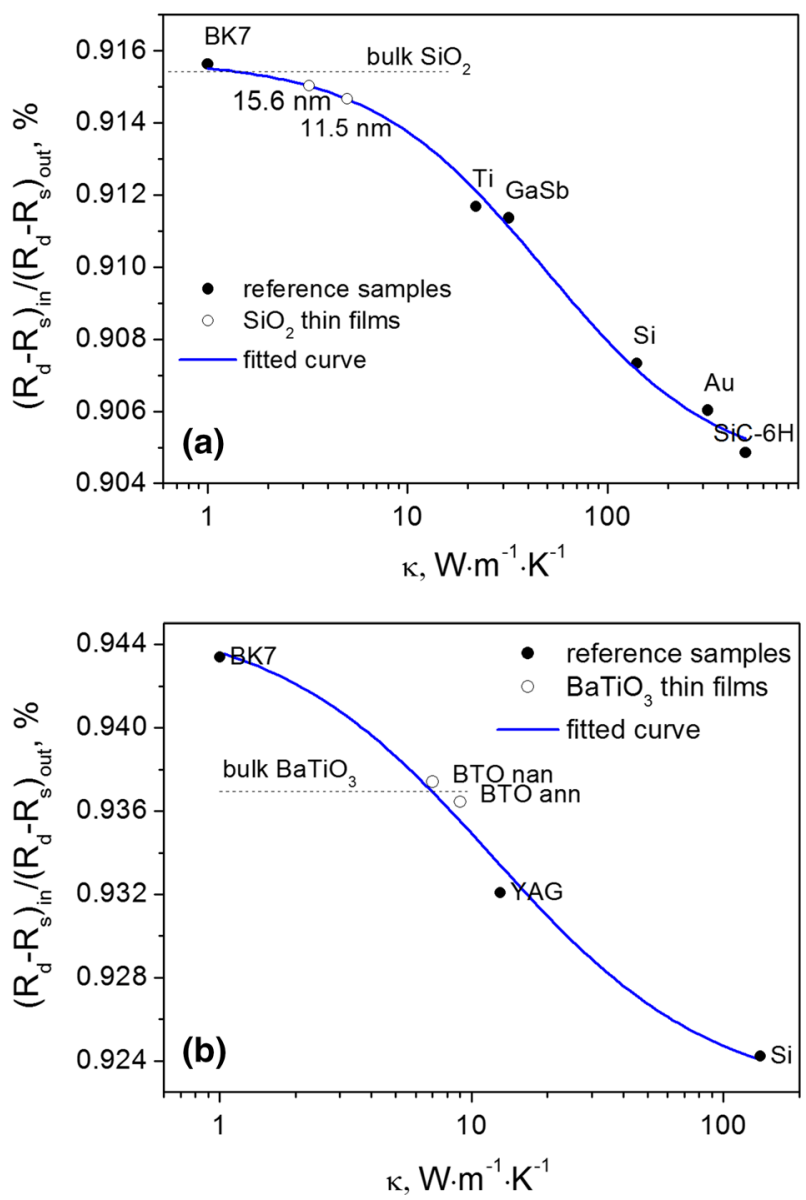

Fig. 8 Results of SThM measurements for (a) thin $\mathrm{SiO}_{2}$ films and (b) thin $\mathrm{BaTiO}_{3}$ films: measured samples $\left({ }^{\circ}\right)$, reference samples $(\bullet)$, and fitted curves $(-)$ described by the Eq. 5 used for estimation of apparent values of thermal conductivity. Dotted line indicates the calculated SThM signal value corresponding to the bulk $\mathrm{SiO}_{2}$ thermal conductivity value of $1.38 \mathrm{~W} \cdot \mathrm{m}^{-1} \cdot \mathrm{K}^{-1}[54]$ and bulk $\mathrm{BaTiO}_{3}$ thermal conductivity value of $4.6 \mathrm{~W} \cdot \mathrm{m}^{-1} \cdot \mathrm{K}^{-1}$

where $A=4 r R_{\mathrm{thP}}$ and $B=A+4 r / h$ (it was assumed that for the probe in air $R_{\text {thP }} \rightarrow \infty$ ). The solid lines in Fig. 8 are the best fit of Eq. 6 with an added constant to reference data points. The constant describes additional convection loses through the tip apex for the probe in air. In the next step, the fitted curve was used to determine the apparent thermal conductivities of layered samples from $\left.R_{\mathrm{th}}\right|_{\text {in }} /\left.R_{\mathrm{th}}\right|_{\text {out }}$ ratio (hollow circles in Fig. 8). The obtained values are listed in the second column of Table 2. Table 2 presents the apparent thermal conductivity values of measured samples. These values are estimated on the basis of the calibration curve constructed from the measurements performed for investigated samples and reference materials. In case of thin films, these values are disrupted by the influence of thermal properties of the substrate. Because of the substrate influence, apparent conductivities directly obtained from 
Table 2 Apparent and corrected thermal conductivity values of investigated thin $\mathrm{SiO}_{2}$ and $\mathrm{BaTiO}_{3}$ layers.

\begin{tabular}{lll}
\hline Thin film & $\begin{array}{l}\text { Apparent thermal } \\
\text { conductivity, W.m }\end{array}$ & $\begin{array}{l}\text { Corrected thermal } \\
\text { conductivity, } \mathrm{W} \cdot \mathrm{K}^{-1} \cdot \mathrm{K}^{-1}\end{array}$ \\
\hline $\mathrm{SiO}_{2} 15.6 \mathrm{~nm}$ & 3.2 & 0.6 \\
$\mathrm{SiO}_{2} 11.5 \mathrm{~nm}$ & 4.9 & 0.5 \\
$\mathrm{BaTiO}_{3}$ annealed & 9.0 & 5.3 \\
$\mathrm{BaTiO}_{3}$ non-annealed & 7.0 & 4.1 \\
\hline
\end{tabular}

Fig. 9 Corrected thermal conductivity values for $\mathrm{SiO}_{2}$ layers $(\bullet)$ versus layer thickness compared with experimental data presented in Refs [55-60] $\left({ }^{\circ}\right)$ and effective thermal conductivity BTE model fitted to the data $(-)$

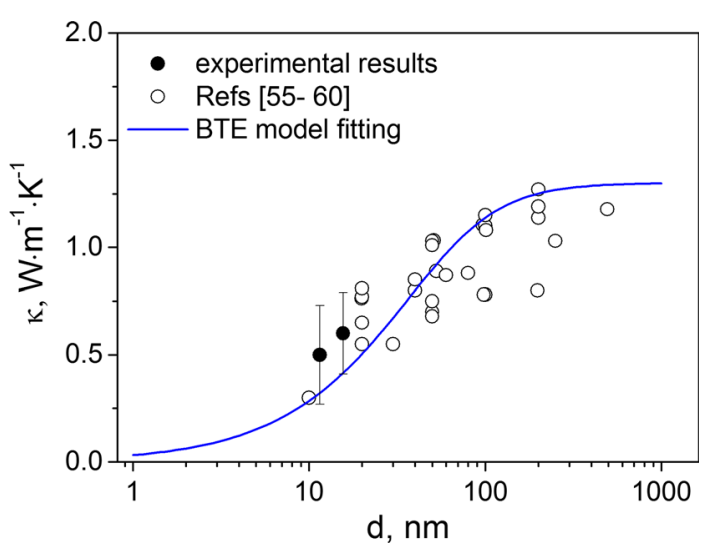

calibration curve are higher than that for bulk materials. Also, apparent conductivity values increase with decreasing layer thickness. A similar effect was observed in [53] for apparent thermal conductivity values, taken from a direct SThM measurement of thin amorphous silicon films, while considered as bulk materials. To estimate the actual thermal conductivity of a thin film, a correction procedure has to be performed on the apparent thermal conductivity values. In the last step, the actual value of the thermal conductivity of the layer was deduced using the procedure described above (the third column in Table 2).

Results obtained for $\mathrm{BaTiO}_{3}$ samples confirm earlier conclusions [52], based on the analysis of probe phase signal, regarding the influence of the manufacturing process on their thermal properties. The actual thermal conductivities obtained for $\mathrm{SiO}_{2}$ samples are compliant with those obtained by other researchers (Fig. 9) [55-60]. The results obtained in this work and accessible in literature can be described by the BTE model of the effective thermal conductivity. The solid line in Fig. 8 is the best fit to the experimental data based on Eq. 1.

The heat transport in investigated layers can still be described classically by Fourier's law. In the case of thinner layers, a transition from diffusive to ballistic heat transport can be expected. Therefore predictions based on Fourier's law can lead to incorrect conclusions. In the ballistic heat transport, the majority of phonons fly across the layer without scattering. The thinner the layer, the higher probability of such process. Very thin layer is practically transparent to phonons so the heat abstrac- 
tion from the thermal probe tip is limited by the thermal conductivity of the substrate. As a result, the actual thermal conductivity should approach this value. The next problem is a continuity of very thin films. In the case of a non-continuous layer, the contact area could embrace regions where direct contact between the probe and the substrate occurs. In this case, there are two parallel channels of the heat transport to the sample - through the thin film to the substrate and direct heat flux to the substrate. With growing contribution of the second channel the effective thermal conductivity should approach the value for the substrate.

\section{Conclusions}

It is shown that application of NThPs for SThM measurements can be used for quantitative thermal measurements. Because of relatively low sensitivity of the probe voltage on the thermal conductivity of the sample, measuring techniques providing good signal-to-noise ratio and being immune to changes of environmental conditions must be developed. One of them can be the technique based on simultaneous determination of the static and the dynamic resistances of the NThP driven by the sum of dc and ac currents, presented in this paper.

To analyze the possibility of determination of the thermal conductivity of thin film deposited on thick substrate from SThM measurement, the numerical model based on FEM was built. This model allows a comprehensive analysis of processes occurring in the SThM probe-sample system. Simulations of SThM thin film measurements were carried out and the influence of the thermal properties of the substrate on the measured signal was investigated. The strongest substrate influence on SThM signal was observed for layers with thicknesses smaller than $1 \mu \mathrm{m}$. The limit thickness, for which the substrate influence disappears, is strongly related to the difference between the thermal conductivities of the layer and the substrate. Results from the simulation allowed for the correction procedure to be determined, which provides the possibility for determining the corrected thermal conductivity of the layer from its apparent value. It was shown that this procedure gives reasonable results for the investigated $\mathrm{SiO}_{2}$ and $\mathrm{BaTiO}_{3}$ samples. The procedure can be used for layers of thicknesses comparable to, or higher than the mean free path of heat carriers, i.e., when the heat transport can still be properly described by the classical equations, on which the procedure was based.

Acknowledgments The support of the PHC Polonium 2015 Project No. 33564VE is greatly acknowledged.

Open Access This article is distributed under the terms of the Creative Commons Attribution 4.0 International License (http://creativecommons.org/licenses/by/4.0/), which permits unrestricted use, distribution, and reproduction in any medium, provided you give appropriate credit to the original author(s) and the source, provide a link to the Creative Commons license, and indicate if changes were made.

\section{References}

1. G. Cahill, A. Bullen, S.M. Lee, High Temp. High Press. 32, 135 (2000)

2. S. Orain, Y. Schudeller, T. Brousse, Int. J. Therm. Sci. 39, 537 (2000)

3. M. Asheghi, K. Kurabayashi, R. Kasnavi, K.E. Goodson, J. Appl. Phys. 91, 5079 (2002)

4. D.M. Bhusari, C.W. Teng, K.H. Chen, S.L. Wei, L.C. Chen, Rev. Sci. Instrum. 68, 4180 (1997) 
5. S. Dilhaire, S. Grauby, W. Clayes, JCh. Batsale, Microelectron. J. 35, 811 (2004)

6. T. Tong, A. Majumdar, Rev. Sci. Instrum. 77, 104902 (2006)

7. B. Li, J.P. Roger, L. Pottier, D. Fournier, J. Appl. Phys. 86, 5314 (1999)

8. A. Kusiak, J.L. Battaglia, S. Gomez, J.P. Manaud, Y. Lepetitcorps, Eur. Phys. J. Appl. Phys. 35, 17 (2006)

9. E. Bozorg-Grayeli, A. Sood, M. Asheghi, V. Gambin, R. Sandhu, T.I. Feygelson, B.B. Pate, K. Hobart, K.E. Goodson, Appl. Phys. Lett. 102, 111907 (2013)

10. D. Cahill, W. Ford, K. Goodson, G. Mahan, A. Majumdar, H. Maris, R. Merlin, S. Phillpot, J. Appl. Phys. 93, 793 (2003)

11. D. Cahill, P. Braun, C. Chen, D. Clarke, S. Fan, K. Goodson, P. Keblinski, W. King, G. Mahan, A. Majumdar, H. Maris, S. Phillpot, E. Pop, L. Shi, Appl. Phys. Rev. 1, 011305 (2014)

12. R. Meinders, J. Mater. Res. 16, 2530 (2001)

13. D. Cahill, K. Goodson, A. Majumdar, J. Heat Transf. 124, 223 (2002)

14. A. Joshi, A. Majumdar, J. Appl. Phys. 74, 31 (1993)

15. M. Xu, L. Wang, Int. J. Heat Mass Trans. 48, 5616 (2005)

16. S. Saha, L. Shi, J. Appl. Phys. 101, 074304 (2007)

17. J. Lukes, X. Liang, C. Tien, D. Li, J. Heat Transf. 122, 536 (2000)

18. R.A. Escobar, C.H. Amon, J. Heat Transf. 130, 092402 (2008)

19. W. Liu, M. Asheghia, Appl. Phys. Lett. 84, 3819 (2004)

20. C. Jeong, S. Datta, M. Lundstrom, J. Appl. Phys. 111, 093708 (2012)

21. N. Donmezer, S. Graham, Int. J. Therm. Sci. 76, 235 (2014)

22. T.-K. Hsiao, H.-K. Chang, S.-C. Liou, M.-W. Chu, S.-C. Lee, C.-W. Chang, Nat. Nanotechnol. 8, 534 (2013)

23. F.X. Alvarez, D. Jou, J. Appl. Phys. 103, 094321 (2008)

24. M. Xu, H. Hu, Proc. R Soc. A 467, 1851 (2011)

25. F.X. Alvarez, D. Jou, Appl. Phys. Lett. 90, 083109 (2007)

26. L. Shi, A. Majumdar, in Applied Scanning Probe Methods, ed. by B. Bhushan, H. Fuchs, M. Tomitoti (Springer, Berlin, 2004), pp. 327-362

27. M. Chirtoc, J.S. Antoniow, J.F. Henry, P. Dole, J. Pelzl, in Advanced Techniques and Applications on Scanning Probe Microscopy, ed. by J.L. Bubendorff, F.H. Lei (Transworld Research Network, Trivandrum, Kerala, 2008), pp. 197-247

28. K. Park, H. Nair, A. Crook, S. Bank, E. Yu, Appl. Phys. Lett. 102, 061912 (2013)

29. J. Chung, K. Kim, G. Hwang, O. Kwon, Int. J. Therm. Sci. 62, 109 (2012)

30. M. Muñoz Rojo, S. Grauby, J.M. Rampnoux, O. Caballero-Calero, M. Martin-Gonzalez, S. Dilhaire, J. Appl. Phys 113, 054308 (2013)

31. E. Puyoo, S. Grauby, J.M. Rampnoux, E. Rouvière, S. Dilhaire, Rev. Sci. Instrum. 81, 073701 (2010)

32. S. Gomes, L. David, V. Lysenko, A. Descamps, T. Nychyporuk, M. Raynaud, J. Phys. D 40, 6677 (2007)

33. S. Gomes, P. Newby, B. Canut, K. Termentzidis, O. Marty, L. Frechette, P. Chantrenne, V. Aimez, J.-M. Bluet, V. Lysenko, Microelectron. J. 44, 1029 (2013)

34. S. Rossi, M. Alomari, Y. Zhang, S. Bychikhin, D. Pogany, J.M.R. Weaver, E. Kohn, Diam. Relat. Mater. 40, 69 (2013)

35. S. Volz, X. Feng, C. Fuentes, P. Guérin, M. Jaouen, Int. J. Thermophys. 23, 1645 (2002)

36. F. Ruiz, W.D. Sun, F.H. Pollak, C. Venkatraman, Appl. Phys. Lett. 73, 1802 (1998)

37. S. Gomès, A. Assy, P.O. Chapuis, Phys. Status Solidi A 212, 477 (2015)

38. S. Lefevre, S. Volz, J.B. Saulnier, C. Fuentes, N. Trannoy, Rev. Sci. Instrum. 74, 2418 (2003)

39. J. Juszczyk, M. Wojtol, J. Bodzenta, Int. J. Thermophys. 34, 620 (2013)

40. H. Fischer, Thermochim. Acta 425, 69 (2005)

41. S. Lefèvre, S. Volz, Rev. Sci. Instrum. 76, 033701 (2005)

42. L. David, S. Gomes, M. Raynaud, J. Phys. D 40, 4337 (2007)

43. P. Tovee, M. Pumarol, D. Zeze, K. Kjoller, O. Kolosov, J. Appl. Phys. 112, 114317 (2012)

44. Y. Joliff, L. Belec, J.-F. Chailan, J. Surf. Eng. Mater. Adv. Technol. 1, 1 (2011)

45. B. Cavallier, S. Ballandras, B. Cretin, P. Vairac, Sensor. Actuat. A Phys. 123, 444 (2005)

46. A. Altes, R. Heiderhoff, L.J. Balk, J. Phys. D 37, 952 (2004)

47. A. Altes, R. Tilgner, W. Walter, Microelectron. Reliab. 46, 1525 (2006)

48. J. Bodzenta, A. Kaźmierczak-Bałata, M. Lorenc, J. Juszczyk, Int. J. Thermophys. 31, 150 (2010)

49. J. Bodzenta, M. Chirtoc, J. Juszczyk, J. Appl. Phys. 116, 054501 (2014) 
50. J. Bodzenta, J. Juszczyk, M. Chirtoc, Rev. Sci. Instrum. 84, 093702 (2013)

51. M.M. Yovanovich, E.E. Marotta, in Heat Transfer Handbook, ed. by A. Bejan, A.D. Kraus (Wiley, New Jersey, 2003), p. 261

52. A. Kaźmierczak-Bałata, J. Bodzenta, M. Krzywiecki, J. Juszczyk, J. Szmidt, P. Firek, Thin Solid Films $\mathbf{5 4 5 ,} 217$ (2013)

53. S. Volz, X. Feng, C. Fuentes, P. Guerin, M. Jaonen, Int. J. Thermophys. 23, 1645 (2001)

54. A.L. Edwards, Compilation of Thermal Property Data for Computer Heat-Conduction Calculations, UCRL_50589 (Lawrence Radiation Lab., Califormia University, Livermore, 1969)

55. R. Kato, I. Hatta, Int. J. Thermophys. 26, 179 (2005)

56. R. Kato, A. Maesono, R.P. Tye, Int. J. Thermophys. 22, 617 (2001)

57. M.G. Burzo, P.L. Komarov, P.E. Raad, IEEE Trans. Compon. Packag. Technol. 26, 80 (2003)

58. T. Yamane, N. Nagai, S.-I. Katayama, M. Todoki, J. Appl. Phys. 91, 9772 (2002)

59. J.H. Kim, A. Feldman, D. Novotny, J. Appl. Phys. 86, 3959 (1999)

60. O.W. Käding, H. Skurk, K.E. Goodson, Appl. Phys. Lett. 65, 1629 (1994) 